\title{
Pacific
}

Journal of

Mathematics

\section{HOMOLOGICAL STABILITY OF SERIES OF GROUPS}

Tim D. Cochran AND Shelly HaRvey 


\title{
HOMOLOGICAL STABILITY OF SERIES OF GROUPS
}

\author{
Tim D. COCHRAN AND Shelly Harvey
}

\begin{abstract}
We define the stability of a subgroup under a class of maps, and establish its basic properties. Loosely speaking, we will say that a normal subgroup, or more generally a normal series $\left\{A_{n}\right\}$ of a group $A$, is stable under a class of homomorphisms $\mathscr{H}$ if whenever $f: A \rightarrow B$ lies in $\mathscr{H}$, we have $f(a) \in B_{n}$ if and only if $a \in A_{n}$. This translates to saying that each element of $\mathscr{H}$ induces a monomorphism $A / A_{n} \hookrightarrow B / B_{n}$. This contrasts with the usual theories of localization, wherein one is concerned with situations where $f$ induces an isomorphism. In the literature, the most commonly considered classes of maps are those that induce isomorphisms on (low-dimensional) group homology. The model theorem in this regard is the result of Stallings that each term of the lower central series is preserved under any $\mathbb{Z}$-homological equivalence of groups. Various other theorems of this nature have since appeared, involving variations of the lower central series. Dwyer generalized Stallings's $\mathbb{Z}$ results to larger classes of maps, work that was completed in the other cases by the authors. More recently, the authors proved analogues of the theorems of Stallings and Dwyer for variations of the derived series. We interpret all of the theorems above in the framework of stability.
\end{abstract}

\section{Introduction}

Loosely speaking, we say that a function $\Gamma$ that assigns to each group $A$ a normal subgroup $\Gamma(A) \triangleleft A$ is stable under a class of maps $\mathcal{H}$ if whenever $f: A \rightarrow B$ lies in $\mathscr{H}$, we have $f(a) \in \Gamma(B)$ if and only if $a \in \Gamma(A)$. Precise definitions are given in Section 2. This translates to saying that each element of $\mathscr{H}$ induces a monomorphism $A / \Gamma(A) \hookrightarrow B / \Gamma(B)$. This contrasts with the usual theories of localization, wherein one is concerned with situations where $f$ induces an isomorphism. The stabilization $\Gamma_{S}$ of $\Gamma$ under $\mathcal{H}$ is the function assigning the "smallest" normal subgroup that contains $\Gamma(A)$ and has the property that each $f \in \mathcal{H}$ induces a monomorphism $A / \Gamma_{S}(A) \hookrightarrow B / \Gamma_{S}(B)$.

MSC2000: 20J05, 57M05.

Keywords: homology equivalence, derived series, groups.

Both authors were partially supported by the National Science Foundation. Harvey was partially supported by a fellowship from the Sloan Foundation and by an NSF CAREER grant. 
We are motivated by the general question, What subgroups of a group are unchanged, or stable, under homology equivalences? The model theorem in this regard is the landmark result of Stallings that each term of the lower central series of a group $A$ is preserved under any homological equivalence. Recall that $A_{n}$ is defined recursively by $A_{1} \equiv A$ and $A_{n+1}=\left[A, A_{n}\right]$.

Stallings's integral theorem [1965, Theorem 3.4]. Suppose $f: A \rightarrow B$ is a group homomorphism that induces an isomorphism on $H_{1}(\cdot ; \mathbb{Z})$ and an epimorphism on $H_{2}(\cdot ; \mathbb{Z})$. Then for each $n, f$ induces an isomorphism $A / A_{n} \cong B / B_{n}$. Therefore $a \in A_{n}$ if and only if $f(a) \in B_{n}$.

This theorem implies that each term of the lower central series is stable under the set of $\mathbb{Z}$-homologically 2 -connected maps.

Stallings had analogous theorems [1965, Theorem 7.3] for $\mathbb{Q}$ and $\mathbb{Z}_{p}$ (here $\mathbb{Z}_{p}$ denotes the integers modulo $p$ ) that involved different series - variations of the lower-central series. Dwyer [1975, Theorem 1.1] improved on Stallings's theorem by weakening the hypothesis on $\mathrm{H}_{2}$ and finding the precise class of maps $f$ that, for a fixed $n$, yield isomorphisms modulo the $n$-th term of the lower central series. This was placed in a larger context by Bousfield [1977].

Dwyer's work implies that the lower central series is stable under a larger class of maps. However, the lower central series fails to be stable if one considers $\mathbb{Q}$ homologically 2 -connected maps, as may be seen by considering the map $\mathbb{Z}_{2} \rightarrow\{e\}$. This situation is remedied by enlarging the lower central series slightly to form the rational lower central series; see Section 2. The rational lower central series is stable under rational homology equivalences, as a consequence of Stallings's rational theorem; see Section 3.

More recently, we found analogues of the theorems of Stallings and Dwyer for the derived series [Cochran and Harvey 2005; 2008a]. However, to do so it was necessary to expand the derived series and use a larger series, the torsion-freederived series, introduced in [Harvey 2008, Section 2]. All of these theorems and series will be reviewed as needed later. The derived series fails dramatically to have the stability property under homological equivalences. For example, if we consider the abelianization map

$$
\mathbb{Z}\left[t, t^{-1}\right] /\left\langle t^{2}-t+1\right\rangle \rtimes \mathbb{Z} \equiv A \stackrel{f}{\longrightarrow} B \equiv \mathbb{Z},
$$

we see that it is a homologically 2-connected map. However, any nonzero element of the commutator subgroup of $A$, that is, $\mathbb{Z}\left[t, t^{-1}\right] /\left\langle t^{2}-t+1\right\rangle$, is not itself in the second derived subgroup of $A$, yet maps trivially under $f$ and hence lies in the second term of the derived series of $\mathbb{Z}$. Can the $n$-th term of the derived series be enlarged until it is stable under homologically 2-connected maps? In this case, expanding to the rational derived series fails. Certainly, this $n$-th term can be so 
enlarged, since we could enlarge it all the way to the $2^{n}$-th term of the lower-central series, which is stable under 2-connected maps. What is the minimum by which it needs to be enlarged to become stable? These questions motivate the definitions to follow.

\section{Stability of series of groups}

Let $\mathscr{G}$ be the category of groups, and let $\mathscr{C}$ be a subcategory.

Definition 2.1. A subgroup function (or normal subgroup function) for $\mathscr{C}$ is a function $\Gamma: \mathscr{C} \rightarrow \mathscr{C}$ that assigns to each $A \in \mathscr{C}$ a normal subgroup $\Gamma(A)$ of $A$.

Definition 2.2. A series $\left\{\Gamma^{n}\right\}$ (or normal series) for $\mathscr{C}$ is a collection of subgroup functions $\Gamma^{n}: \mathscr{C} \rightarrow \mathscr{C}$, with $n \geq 0$, such that

$$
\{e\} \subset \cdots \subset \Gamma^{n+1}(A) \subset \Gamma^{n}(A) \cdots \subset \Gamma^{1}(A) \subset \Gamma^{0}(A)=A .
$$

The important examples to keep in mind are the lower central series $\left\{A_{n}\right\}$, the rational lower central series, $\left\{A_{n}^{r}\right\}$, defined by

$$
A_{1}^{r}=A, \quad A_{n+1}^{r}=\left\{x \mid x^{k} \in\left[A, A_{n}^{r}\right] \text { for some positive integer } \mathrm{k}\right\},
$$

and, for a fixed prime $p$, the $\mathbb{Z}_{p}$-lower central series $\left\{A_{p, n}\right\}$ (also called the $p$-lower central series or the lower central $p$-series), which is defined by

$$
A_{p, 1}=A, \quad A_{p, n+1}=\left(A_{p, n}\right)^{p}\left[A_{p, n}, A\right] .
$$

This is the fastest-descending central series whose successive quotients are $\mathbb{Z}_{p^{-}}$ vector spaces [Stallings 1965].

Definition 2.3. For $R=\mathbb{Z}, \mathbb{Q}$, or $\mathbb{Z}_{p}$, where $p$ is prime, let the $R$-lower central series $\left\{A_{n}^{R}\right\}$ be the lower central series, rational lower central series, or $\mathbb{Z}_{p}$-lower central series, respectively.

The derived series $\left\{A^{(n)}\right\}$ is given by

$$
A^{(0)}=A, \quad A^{(n+1)}=\left[A^{(n)}, A^{(n)}\right] ;
$$

the rational derived series $\left\{A_{r}^{(n)}\right\}$ is defined by

$$
A^{(0)}=A, \quad A_{r}^{(n+1)}=\left\{x \mid x^{k} \in\left[A_{r}^{(n)}, A_{r}^{(n)}\right] \text { for some positive integer k }\right\} ;
$$

and the $\mathbb{Z}_{p}$-derived series $\left\{A_{p}^{(n)}\right\}$ (also called the $p$-derived series or the derived $p$-series) is defined by

$$
A_{p}^{(0)}=A, \quad A_{p}^{(n+1)}=\left(A_{p}^{(n)}\right)^{p}\left[A_{p}^{(n)}, A_{p}^{(n)}\right] .
$$

This is the fastest-descending series whose successive quotients are $\mathbb{Z}_{p}$-vector spaces [Stallings 1965]. 
Definition 2.4. A class of maps $\mathscr{H}$ for $\mathscr{b}$ is a subset of the morphisms of $\mathscr{C}$ that contains all isomorphisms, is closed under composition, and is closed under nudgeouts, by which we mean that if $f, f^{\prime} \in \mathscr{H}$, as in the diagram below, then there exist $g$ and $g^{\prime}$ in $\mathscr{H}$ that make the following diagram commute:

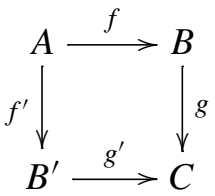

Clearly, being closed under push-outs implies being closed under nudge-outs.

Definition 2.5. A subgroup function $\Gamma$ is $\mathscr{H}$-invariant with respect to the class of maps $\mathscr{H}$ if $f(\Gamma(A)) \subset \Gamma(B)$ whenever $f: A \rightarrow B$ is an element of $\mathscr{H}$. (This is the same as saying that, for each $n, \Gamma$ is functorial on the category $\mathscr{C}$ with morphisms restricted to lie in $\mathscr{H}$.) A series $\left\{\Gamma^{n} \mid n \geq 0\right\}$ is $\mathscr{H}$-invariant with respect to a collection of classes of maps $\mathscr{H}=\left\{\mathscr{H}^{n} \mid n \geq 0\right\}$ satisfying

$$
\cdots \subseteq \mathscr{H}^{n} \subseteq \cdots \subseteq \mathscr{H}^{1} \subseteq \mathscr{H}^{0} \subset \operatorname{Morph}(\mathscr{C})
$$

if $f\left(\Gamma^{n}(A)\right) \subset \Gamma^{n}(B)$ whenever $f: A \rightarrow B$ is an element of $\mathscr{H}^{n}$.

Remark 2.6. Each of the versions of the lower central series and derived series defined above, consisting (essentially) of verbal subgroups, is $\mathscr{H}$-invariant with respect to any class of maps.

Definition 2.7. Suppose $\Gamma$ is an $\mathscr{H}$-invariant subgroup function. The stabilization of $\Gamma$ with respect to $\mathscr{H}$, denoted $\Gamma_{S}$, is the subgroup

$$
\Gamma_{S}(A)=\{a \in A \mid \text { there exists } f: A \rightarrow B, f \in \mathscr{H} \text {, such that } f(a) \in \Gamma(B)\} .
$$

We say that $\Gamma$ is stable under $\mathscr{H}$ if $\Gamma_{S}(A)=\Gamma(A)$ for each $A$ in $\mathscr{C}$. Suppose $\left\{\Gamma^{n}\right\}$ is an $\mathscr{H}$-invariant series. The stabilization $\left\{\Gamma_{S}^{n}\right\}$ of $\left\{\Gamma^{n}\right\}$ with respect to $\mathscr{H}=\left\{\mathscr{H}^{n}\right\}$ is the series wherein $\Gamma_{S}^{n}$ is the stabilization of $\Gamma^{n}$ with respect to $\mathscr{H}^{n}$. We say that the series $\left\{\Gamma^{n}\right\}$ is stable under $\mathscr{H}$ if each term is stable under the corresponding class of maps.

Remark 2.8. If $\mathscr{H} \subset \widetilde{\mathscr{H}}$, then clearly $\Gamma_{S, \mathscr{H}} \subset \Gamma_{S, \widetilde{\mathscr{H}}}$.

We first verify that $\Gamma_{S}$ is itself a subgroup function.

Proposition 2.9. If $\Gamma$ is an $\mathscr{H}$-invariant subgroup function, $\Gamma_{S}$ is an H-invariant subgroup function. If $\left\{\Gamma^{n}\right\}$ is an $\mathscr{H}$-invariant series, $\left\{\Gamma_{S}^{n}\right\}$ is an $\mathscr{H}$-invariant series.

Proof. First we show that $\Gamma_{S}(A)$ is a normal subgroup of $A$. Clearly the identity $e$ lies in $\Gamma_{S}(A)$. Let $a, a^{\prime} \in \Gamma_{S}(A)$, so there exist $f, f^{\prime} \in \mathscr{H}$ such that $f(a) \in \Gamma(B)$ 
and $f^{\prime}\left(a^{\prime}\right) \in \Gamma\left(B^{\prime}\right)$. Since $\mathscr{H}$ is closed under nudge-outs, there exists $C$, as shown below, with $g, g^{\prime} \in \mathscr{H}^{\text {. }}$

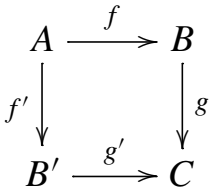

Since $\mathscr{H}$ is closed under composition, $g \circ f=g^{\prime} \circ f^{\prime} \in \mathscr{H}$. Since $\Gamma$ is $\mathscr{H}$-invariant, we also have

$$
g^{\prime}\left(f^{\prime}\left(a^{\prime}\right)\right) \subset g^{\prime}\left(\Gamma\left(B^{\prime}\right)\right) \subset \Gamma(C) \quad \text { and } \quad g(f(a)) \subset g(\Gamma(B)) \subset \Gamma(C) .
$$

Thus,

$$
(g \circ f)\left(a a^{\prime}\right)=(g \circ f)(a) \cdot(g \circ f)\left(a^{\prime}\right)=g(f(a)) \cdot g^{\prime}\left(f^{\prime}\left(a^{\prime}\right)\right),
$$

implying that $(g \circ f)\left(a a^{\prime}\right) \in \Gamma(C)$. Thus $a a^{\prime} \in \Gamma_{S}(A)$. Also, if $f(a) \in \Gamma(B)$, then $f\left(a^{-1}\right) \in \Gamma(B)$ since $\Gamma(B)$ is a subgroup, so $\Gamma_{S}$ is closed under taking inverses. Similarly, if $f(a) \in \Gamma(B)$, then $f\left(x a x^{-1}\right)=f(x) f(a)(f(x))^{-1}$ lies in $\Gamma(B)$ since $\Gamma(B)$ is a normal subgroup. Therefore $\Gamma_{S}(A)$ is a normal subgroup of $A$.

Next we show that $\Gamma_{S}$ is $\mathscr{H}$-invariant. Suppose $f: A \rightarrow B, f \in \mathscr{H}$ and $a \in \Gamma_{S}(A)$. Then there exists $f^{\prime} \in \mathscr{H}$ such that $f^{\prime}(a) \in \Gamma\left(B^{\prime}\right)$. Since $\mathscr{H}$ is closed under nudgeouts, there exists $C$ as below with $g$ and $g^{\prime}$ in $\mathscr{H}$ and $g \circ f=g^{\prime} \circ f^{\prime} \in \mathscr{H}$.

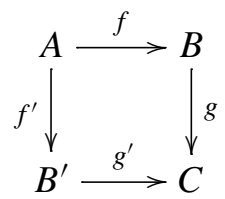

Since $\Gamma$ is $\mathscr{H}$-invariant, $g^{\prime}\left(f^{\prime}(a)\right) \in \Gamma(C)$. Thus $(g \circ f)(a) \in \Gamma(C)$. Since $g \in \mathscr{H}$, it follows that $f(a) \in \Gamma_{S}(B)$ as desired.

Now, suppose that $\left\{\Gamma^{n}\right\}$ is an $\mathscr{H}=\left\{\mathscr{C}^{n}\right\}$-invariant series. First we show that $\Gamma_{S}^{n+1}(A) \subset \Gamma_{S}^{n}(A)$. Suppose that $a \in \Gamma_{S}^{n+1}(A)$, so that there exists $f \in \mathscr{H}^{n+1}$ such that $f(a) \in \Gamma^{n+1}(B)$; then, by our nesting requirement on $\mathscr{H}$, we have $f \in \mathscr{H}^{n}$ and $f(a) \in \Gamma^{n}(B)$, so $a \in \Gamma_{S}^{n}(A)$. Finally, if $\Gamma^{0}(A)=A$, then, since $\Gamma^{0}(A) \subset \Gamma_{S}^{0}(A)$, we have $A=\Gamma^{0}(A)=\Gamma_{S}^{0}(A)$.

Theorem 2.10 (properties of the stabilization of a subgroup function). Suppose $\mathscr{H}$ is a class of maps and $\Gamma$ is an $\mathscr{H}$-invariant subgroup.

(1) For each $A \in \mathscr{C}, \Gamma(A)$ is a normal subgroup of $\Gamma_{S}(A)$.

(2) Every $f: A \rightarrow B$ with $f \in \mathscr{H}$ induces a monomorphism

$$
A / \Gamma_{S}(A) \hookrightarrow B / \Gamma_{S}(B) .
$$


(3) $\Gamma_{S}$ is the initial He-invariant subgroup function satisfying (1) and (2). To be specific, if $\{\widetilde{\Gamma}\}$ is an H-invariant subgroup function such that

(3.1) $\Gamma(A) \subset \widetilde{\Gamma}(A)$ for all $A \in \mathscr{C}$, and

(3.2) every $f \in \mathscr{H}$ induces a monomorphism $A / \widetilde{\Gamma}(A) \hookrightarrow B / \widetilde{\Gamma}(B)$, then $\Gamma_{S}(A) \subset \widetilde{\Gamma}(A)$ for all $A \in \mathscr{C}$.

Proof. Any $x$ in $\Gamma(A)$ is also in $\Gamma_{S}(A)$, since the identity map $i_{A}$ is in $\mathscr{H}$. Normality was established in the proof of Proposition 2.9. This establishes (1).

Suppose $f: A \rightarrow B$, with $f \in \mathscr{H}$. Since $\Gamma_{S}$ is $\mathscr{H}$-invariant by Proposition 2.9, $f$ induces a map $f: A / \Gamma_{S}(A) \rightarrow B / \Gamma_{S}(B)$. Now suppose $f(a) \in \Gamma_{S}(B)$. Then, by definition, there exists a $g: B \rightarrow C$ with $g \in \mathscr{H}$ such that $g \circ f(a) \in \Gamma(C)$. Since $\mathscr{H}$ is closed under composition, $g \circ f \in \mathscr{H}$. Hence $a \in \Gamma_{S}(A)$, establishing (2).

Now suppose $\widetilde{\Gamma}$ is another $\mathscr{H}$-invariant series satisfying (3.1) and (3.2). Suppose that $a \in \Gamma_{S}(A)$, so there exists an $f: A \rightarrow B$ with $f \in \mathscr{H}$ such that $f(a) \in \Gamma(B)$. By property (3.1) for $\widetilde{\Gamma}$, we have $\Gamma(B) \subset \widetilde{\Gamma}(B)$, so $f(a) \in \widetilde{\Gamma}(B)$. By property (3.2) for $\widetilde{\Gamma}, f$ induces a monomorphism $f: A / \widetilde{\Gamma}(A) \hookrightarrow B / \widetilde{\Gamma}(B)$. Hence $a \in \widetilde{\Gamma}(A)$ and $\Gamma_{S}(A) \subset \widetilde{\Gamma}(A)$, establishing (3).

Now we arrive at our major tool for determining the stabilization.

Corollary 2.11. If $\Gamma$ is an $\mathscr{H}$-invariant subgroup function for which every $f \in \mathscr{H}$ induces a monomorphism $A / \Gamma(A) \rightarrow B / \Gamma(B)$, then $\Gamma_{S}(A)=\Gamma(A)$ for all $A \in \mathscr{C}$; that is, $\Gamma$ is stable under $\mathscr{H}$. If $\left\{\Gamma^{n}\right\}$ is an $\mathscr{H}$-invariant series for which every $f \in \mathscr{H}^{n}$ induces a monomorphism $A / \Gamma^{n}(A) \rightarrow B / \Gamma^{n}(B)$, then $\left\{\Gamma^{n}\right\}$ is stable under $\mathcal{H}$.

Proof. Since $\Gamma$ satisfies (3.1) and (3.2) above, $\Gamma_{S}(A) \subset \Gamma(A)$. By property (1) of Theorem 2.10, $\Gamma(A) \subset \Gamma_{S}(A)$.

Corollary 2.12 (idempotency). The stabilization of an He-invariant subgroup function (or series), with respect to $\mathscr{H}$, is itself stable with respect to $\mathscr{H}$.

Proof. By Proposition 2.9 and part (2) of Theorem 2.10, $\Gamma_{S}$ satisfies the hypothesis of Corollary 2.11. Thus $\Gamma_{S}$ is itself stable under $\mathscr{H}$.

\section{Stabilizations of the $\boldsymbol{R}$-lower central series}

Let $R=\mathbb{Z}, \mathbb{Q}$ or $\mathbb{Z}_{p}$, where $p$ is prime. Suppose $\mathscr{C}$ is the category of all groups and consider $\mathscr{H}=\mathscr{H}^{R}$, the set of all homologically 2-connected maps with $R$ coefficients, that is, homomorphisms that induce isomorphisms on $H_{1}(\cdot ; R)$ and epimorphisms on $H_{2}(\cdot ; R)$. It is an easy exercise to show that these classes are closed under push-outs. Let $\Gamma^{n} A=A_{n+1}^{R}$; that is, $\Gamma^{n}$ is the $(n+1)$-st term of the $R$-lower central series. Results of Stallings establish that maps in $\mathscr{H}^{R}$ induce monomorphisms modulo any term of the $R$-lower central series (for $R=\mathbb{Z}$ see Stallings's integral theorem; for $R=\mathbb{Q}$ see [Stallings 1965, Theorem 7.3]; and for 
$R=\mathbb{Z}_{p}$ see [Stallings 1965, Theorem 3.4]). Therefore, using Corollary 2.11, these can be reinterpreted as follows:

Proposition 3.1. The $R$-lower central series is stable with respect to all 2-connected maps with $R$-coefficients; that is, $\Gamma_{S}^{n}(A)=\Gamma^{n}(A)$ under $\mathscr{H}^{R}$.

We will show that the $R$-lower central series is stable under a much larger class of maps than $\mathscr{H}^{R}$. The work of Dwyer suggests the following filtration of $H_{2}(A ; R)$.

Definition 3.2. For any group $A$ and positive integer $n$, and for $R=\mathbb{Z}, \mathbb{Q}$ or $\mathbb{Z}_{p}$, let $\Phi_{n}^{R}(A)$ denote the kernel of the natural map

$$
H_{2}(A ; R) \rightarrow H_{2}\left(A / A_{n} ; R\right) .
$$

Note that if $m \geq n$, then $\Phi_{m}^{R}(A) \subset \Phi_{n}^{R}(A)$.

This in turn suggests the following class of maps.

Definition 3.3. For $R=\mathbb{Z}, \mathbb{Q}$ or $\mathbb{Z}_{p}$, let $\mathscr{H}_{\text {Dwyer }}^{R} \equiv\left\{\left(\mathcal{H}_{\text {Dwyer }}^{R}\right)^{n}\right\}$ be the class of maps, called the Dwyer R-class: A map is in $\left(\mathscr{H}_{\text {Dwyer }}^{R}\right)^{n}$ if it induces an isomorphism on $H_{1}(\cdot ; R)$ and an epimorphism

$$
H_{2}(A ; R) / \Phi_{n}^{R}(A) \rightarrow H_{2}(B ; R) / \Phi_{n}^{R}(B) .
$$

One easily checks that these classes are closed under push-outs.

Theorem 3.4. The R-lower central series is stable with respect to Dwyer's $R$-class of maps, $\mathcal{H}_{\text {Dwyer }}^{R}$.

Proof. By Remark 2.6, we see that the $R$-lower central series $\Gamma^{n}(A)=A_{n+1}^{R}$ is $\mathscr{H}_{\text {Dwyer }}^{R}$-invariant. By Corollary 2.11, it suffices to show that any map $f: A \rightarrow B$ such that $f \in\left(\mathcal{H}_{\text {Dwyer }}^{R}\right)^{n}$ induces a monomorphism

$$
A / A_{n+1}^{R} \rightarrow B / A_{n+1}^{R} .
$$

In the case $R=\mathbb{Z}$, this was established by Dwyer [1975, Theorem 1.1]. More recently, we showed this in the cases $R=\mathbb{Q}$ and $R=\mathbb{Z}_{p}[\mathrm{CH} 2008 \mathrm{a}$, Theorem 3.1; 2008b, Theorem 3.1].

Despite these positive results there are simple unanswered questions:

Question 3.5. Is the stabilization of the lower central series under $\mathscr{H}^{R}$ (for $R=\mathbb{Z}_{p}$ or $\mathbb{Q}$ ) equal to the $R$-lower central series?

We do not know the answer to this question. However:

Proposition 3.6. The stabilization of the lower central series under $\mathcal{H}_{\mathrm{Dwyer}}^{R}$ is the $R$-lower central series. More generally, the stabilization under $\mathcal{H}_{\text {Dwyer }}^{R}$ of any series that is contained in the $R$-lower central series is the $R$-lower central series. 
This is the first case we have discussed where the stabilization is strictly larger than the original series and we can calculate the stabilization precisely.

Proof. Suppose $\Gamma^{n}(A) \subset \widetilde{\Gamma}^{n}(A)=A_{n+1}^{R}$. Then property (3.1) of Theorem 2.10 holds. By the theorems [Dwyer 1975, Theorem 1.1]; [CH 2008a, Theorem 3.1]; and [CH 2008b, Theorem 3.1], property (3.2) also holds. So by Theorem 2.10, $\Gamma_{S}^{n}(A) \subset \widetilde{\Gamma}^{n}(A)$.

We must show that $\widetilde{\Gamma}^{n}(A) \subset \Gamma_{S}^{n}(A)$. This is trivially true for $n=0$, so assume that $n>0$. Suppose $a \in \widetilde{\Gamma}^{n}(A)=A_{n+1}^{R}$, and consider the projection $f: A \rightarrow A /\langle a\rangle \equiv B$. Since $f(a)=1, f(a) \in \Gamma^{n}(B)$. Hence, if we can establish that $f \in\left(\mathscr{H}_{\text {Dwyer }}^{R}\right)^{n}$, then $a \in \Gamma_{S}^{n}(A)$ by definition. Since $n+1 \geq 2$, we have $a \in A_{2}^{R}$. Note that

$$
\begin{aligned}
H_{1}\left(A ; \mathbb{Z}_{p}\right) & =A / A_{p, 2}=A / A_{2}^{R} & & \text { when } R=\mathbb{Z}_{p}, \\
H_{1}(A ; \mathbb{Q}) & =A / A_{2}^{r} \otimes \mathbb{Q}=A / A_{2}^{R} \otimes \mathbb{Q} & & \text { when } R=\mathbb{Q} .
\end{aligned}
$$

Thus in all cases it follows that $f$ induces an isomorphism on $H_{1}(\cdot ; R)$. It is fairly easy to show that, since the kernel of $f$ is contained in $A_{n+1}^{R}, f$ induces an isomorphism $A / A_{n+1}^{R} \cong B / A_{n+1}^{R}$. To show that $f \in\left(\mathcal{H}_{\text {Dwyer }}^{R}\right)^{n}$, it now suffices to show that $f$ induces an epimorphism

$$
H_{2}(A ; R) \rightarrow H_{2}(B ; R) / \Phi_{n}^{R}(B) .
$$

In the cases $R=\mathbb{Z}, \mathbb{Q}$, and $\mathbb{Z}_{p}$, this follows from [Dwyer 1975, Theorem 1.1]; [CH 2008a, Theorem 3.1]; and [CH 2008b, Theorem 3.1], respectively, specifically from the converses to the parts of those theorems used above. In the case of [CH 2008b, Theorem 3.1] this is not explicitly stated, but the reader can see that it follows directly from the last commutative diagram of its proof.

\section{The stabilization of the $p$-derived series}

The derived series is not stable under homological equivalences. However, we find that the $p$-derived series behaves more like the lower central series. The underlying reason for this is that if $A$ is finitely generated, then $A / A_{p}^{(n)}$ is a finite $p$-group and hence nilpotent. In this section, let $\Gamma^{n}(A)=A_{p}^{(n)}$.

Proposition 4.1. The $\mathbb{Z}_{p}$-lower central series is stable with respect to all 2-connected maps with $\mathbb{Z}_{p}$-coefficients between finitely generated groups, that is,

$$
\Gamma_{S}^{n}(A)=\Gamma^{n}(A)
$$

under $\mathcal{H}^{\mathbb{Z}_{p}}$ (but restricting to finitely generated groups).

Proof. This follows from Corollary 2.11 and [CH 2008b, Corollary 4.3].

In fact, this recent work of the authors allows us to also show that the $\mathbb{Z}_{p^{-}}$ lower central series is stable with respect to a larger class of maps that induce only 
monomorphisms on $H_{1}\left(\cdot ; \mathbb{Z}_{p}\right)$. This leaves the realm of usual homological localization theory, which is concerned with homological equivalences; see Section 6

Proposition 4.2. The $\mathbb{Z}_{p}$-lower central series is stable with respect to the class of all maps between finitely generated groups that induce a monomorphism on $H_{1}\left(\cdot ; \mathbb{Z}_{p}\right)$ and an epimorphism on $H_{2}\left(\cdot ; \mathbb{Z}_{p}\right)$.

We will now show that the $\mathbb{Z}_{p}$-lower central series is stable under a much larger class of maps. First we define a filtration of $H_{2}\left(A ; \mathbb{Z}_{p}\right)$ analogous to Dwyer's but appropriate for the derived series.

Definition 4.3. For a group $A$ and a nonnegative integer $n$, let $\Phi_{p}^{(n)}(A)$ denote the image of the inclusion-induced map $H_{2}\left(A_{p}^{(n)} ; \mathbb{Z}_{p}\right) \rightarrow H_{2}\left(A ; \mathbb{Z}_{p}\right)$.

This in turn suggests the following class of maps.

Definition 4.4. Let $\mathscr{H}_{p}^{\mathrm{CH}} \equiv\left\{\left(\mathscr{H}_{p}^{\mathrm{CH}}\right)^{n}\right\}$ be the class of maps whose elements are maps $f: A \rightarrow B$, where $A$ is finitely generated, $B$ is finitely presented, and $f$ induces an isomorphism on $H_{1}\left(\cdot ; \mathbb{Z}_{p}\right)$ and an epimorphism

$$
H_{2}\left(A ; \mathbb{Z}_{p}\right) / \Phi_{p}^{(n-1)}(A) \rightarrow H_{2}\left(B ; \mathbb{Z}_{p}\right) / \Phi_{p}^{(n-1)}(B) .
$$

One easily checks that this class is closed under push-outs.

Proposition 4.5. The $\mathbb{Z}_{p}$-lower central series is stable with respect to $\mathscr{H}_{p}^{\mathrm{CH}}$, and in fact is stable with respect to the larger class of maps that induce a monomorphism on $H_{1}\left(\cdot ; \mathbb{Z}_{p}\right)$ rather than an isomorphism.

Proof. This follows from Corollary 2.11 and [CH 2008b, Theorem 4.2].

\section{The stabilization of the derived series}

The derived series and the rational derived series, unlike the lower central series and the $p$-derived series, are highly unstable under homology equivalences. Indeed, until quite recently, nothing much was known or even suspected about their properties under homological equivalences. In 2003, Harvey [2008, Section 2] introduced a superseries $G_{H}^{(n)}$ of the derived series, the torsion-free derived series, for which Cochran and Harvey $[2005 ; 2008$ a] were able to prove analogues of the theorems of Stallings and Dwyer. Here we examine the relationships between the torsion-free derived series and the stabilizations of the ordinary derived series with respect to several natural classes of maps.

We first recall the recursive definition of the torsion-free derived series. Let $G_{H}^{(0)} \equiv G$. Suppose $G_{H}^{(n)}$ has been defined as a normal subgroup of $G$. Then $G_{H}^{(n)} /\left[G_{H}^{(n)}, G_{H}^{(n)}\right]$ is a right $\mathbb{Z}\left[G / G_{H}^{(n)}\right]$-module ( $G$ acts by conjugation). Because $\mathbb{Z}\left[G / G_{H}^{(n)}\right]$ is an Ore domain [Harvey 2008, Proposition 2.1], this module has a well-defined torsion submodule $T$. Then $G_{H}^{(n+1)}$ is defined to be the inverse image 
of $T$ under the natural map $\pi: G_{H}^{(n)} \rightarrow G_{H}^{(n)} /\left[G_{H}^{(n)}, G_{H}^{(n)}\right]$. An easy induction shows that $G^{(n)} \subset G_{r}^{(n)} \subset G_{H}^{(n)}$.

We define several natural classes of maps and then investigate the stabilization of the derived series with respect to these classes. The classes $\mathscr{H}^{\mathbb{Z}}$ and $\mathscr{H}^{\mathbb{Q}}$ have been previously defined as those maps that are homologically 2-connected with coefficients in $\mathbb{Z}$ or $\mathbb{Q}$. In this section we will use these symbols to designate these same classes, but restricted to those 2-connected maps $f: A \rightarrow B$ wherein $A$ is finitely generated and $B$ is finitely presented.

Definition 5.1. For a group $A$ and a nonnegative integer $n$, let $\Phi^{(n)}(A)$ and $\Phi_{\mathbb{Q}}^{(n)}(A)$ denote the images of $H_{2}\left(A^{(n)} ; \mathbb{Z}\right) \rightarrow H_{2}(A ; \mathbb{Z})$ and $H_{2}\left(A^{(n)} ; \mathbb{Q}\right) \rightarrow H_{2}(A ; \mathbb{Q})$, respectively.

The analogy between this filtration and Dwyer's filtration involving the lower central series may not be apparent. See [CH 2008a, Section 1] for a discussion.

Definition 5.2. Define $\mathscr{H}^{\mathrm{CH}} \equiv\left\{\left(\mathscr{H}^{\mathrm{CH}}\right)^{n}\right\}$ (respectively $\left.\mathscr{H}_{\mathbb{Q}}^{\mathrm{CH}} \equiv\left\{\left(\mathscr{H}_{\mathbb{Q}}^{\mathrm{CH}}\right)^{n}\right\}\right)$ to be the class whose elements are maps $f: A \rightarrow B$, where $A$ is finitely generated, $B$ is finitely presented, and $f$ induces an isomorphism on $H_{1}(\cdot ; \mathbb{Z})$ and an epimorphism $H_{2}(A ; \mathbb{Q}) / \Phi^{(n-1)}(A) \rightarrow H_{2}(B ; \mathbb{Z}) / \Phi^{(n-1)}(B)$ (respectively, an isomorphism on $H_{1}(\cdot ; \mathbb{Q})$ and an epimorphism $\left.H_{2}(A ; \mathbb{Q}) / \Phi_{\mathbb{Q}}^{(n-1)}(A) \rightarrow H_{2}(B ; \mathbb{Q}) / \Phi_{\mathbb{Q}}^{(n-1)}(B)\right)$.

One easily checks that these classes are closed under composition and push-outs. It is also clear that $\mathscr{H}^{\mathbb{Z}} \subset \mathscr{H}^{\mathrm{CH}}, \mathscr{H}^{\mathbb{Q}} \subset \mathscr{H}_{\mathbb{Q}}^{\mathrm{CH}}$ and $\mathscr{H}^{\mathrm{CH}} \subset \mathscr{H}_{\mathbb{Q}}^{\mathrm{CH}}$.

The following gives an "upper bound" on the stabilization of the derived series.

Theorem 5.3. Let $\Gamma^{n}(A)=A^{(n)}$, the derived series. Let $\Gamma_{S}^{n}$ be the stabilization of the derived series with respect to $\mathscr{H}_{\mathbb{Q}}^{\mathrm{CH}}$. Then $A^{(n)} \subset \Gamma_{S}^{n}(A) \subset A_{H}^{(n)} \cap A_{2^{n}}^{r}$. The same holds for stabilization with respect to any class of maps contained in $\mathcal{H}_{\mathbb{Q}}^{\mathrm{CH}}$, such as $\mathscr{H}^{\mathbb{Z}}, \mathscr{H}^{\mathbb{Q}}$ and $\mathscr{H}^{\mathrm{CH}}$. (Indeed the same holds if we enlarge the class $\mathscr{H}_{\mathbb{Q}}^{\mathrm{CH}}$ by relaxing the isomorphism condition on $H_{1}$ to a monomorphism condition.)

Proof. It follows from [CH 2008a, Theorem 2.1] that the torsion-free derived series is $\mathscr{H}_{\mathbb{Q}}^{\mathrm{CH}}$-invariant and any $f \in\left(\mathscr{H}_{\mathbb{Q}}^{\mathrm{CH}}\right)^{n}$ induces a monomorphism $A / A_{H}^{(n)} \hookrightarrow B / B_{H}^{(n)}$. Since the torsion-free derived series contains the derived series, we conclude that the torsion-free derived series satisfies (3.1) and (3.2) of Theorem 2.10 (letting $\left.\tilde{\Gamma}^{n}(A)=A_{H}^{(n)}\right)$, and hence the stabilization of the derived series is no larger than the torsion-free derived series, that is, $\Gamma_{S}^{n}(A) \subset A_{H}^{(n)}$.

On the other hand, if we consider the series given by $\tilde{\Gamma}^{n}(A)=A_{2^{n}}^{r}$, the $2^{n}$-th term of the rational lower central series, then we claim that this satisfies (1) and (2) of Theorem 2.10. Once we have shown this, $\Gamma_{S}^{n}(A) \subset \tilde{\Gamma}^{n}(A)=A_{2^{n}}^{r}$.

Now we prove the claim. We will show that $f \in\left(\mathscr{H}_{\mathbb{Q}}^{\mathrm{CH}}\right)^{n}$ implies at once that $f \in\left(\mathscr{H}_{\text {Dwyer }}^{\mathbb{Q}}\right)^{2^{n}-1}$. Then the claim follows directly from [CH 2008a, Theorem 3.1]. 
Let $f \in\left(\mathcal{H}_{\mathbb{Q}}^{\mathrm{CH}}\right)^{n}$. Then by Definitions 5.2 and 5.1, it induces an epimorphism

$$
f_{*}: H_{2}(A ; \mathbb{Q}) \rightarrow H_{2}(B ; \mathbb{Q}) / \Phi_{\mathbb{Q}}^{(n-1)}(B),
$$

and we need to show (by Definitions 3.3 and 3.2) that it induces an epimorphism

$$
f_{*}: H_{2}(A ; \mathbb{Q}) \rightarrow H_{2}(B ; \mathbb{Q}) / \Phi_{2^{n}-1}^{\mathbb{Q}}(B) .
$$

Therefore it suffices to show that $\Phi_{\mathbb{Q}}^{(n-1)}(B) \subset \Phi_{2^{n}-1}^{\mathbb{Q}}(B)$. For this it suffices to show that the composition

$$
H_{2}\left(B^{(n-1)} ; \mathbb{Z}\right) \stackrel{i_{*}}{\longrightarrow} H_{2}(B ; \mathbb{Z}) \stackrel{\pi_{*}}{\longrightarrow} H_{2}\left(B / B_{2^{n}-1} ; \mathbb{Z}\right)
$$

is the zero map. Since $B^{(n-1)} \subset B_{2^{n-1}}$, this follows from Lemma 5.4 below (setting $k=2^{n-1}$, then $2 k-1=2^{n}-1$ ).

Lemma 5.4. For any group $B$ and integer $k$, the map

$$
H_{2}\left(B_{k} ; \mathbb{Z}\right) \stackrel{i_{*}}{\longrightarrow} H_{2}(B ; \mathbb{Z}) \stackrel{\pi_{*}}{\longrightarrow} H_{2}\left(B / B_{2 k-1} ; \mathbb{Z}\right)
$$

is the zero map.

Proof. Suppose $B_{k}$ is presented by $\left\langle F^{\prime} \mid R^{\prime}\right\rangle$. Of course $i\left(F^{\prime}\right) \subset B_{k}$, but we can choose a generating set $F$ for $B$ so large that $i\left(F^{\prime}\right) \subset F_{k}$. Suppose $B$ is presented by $\langle F \mid R\rangle$. It follows that $B / B_{2 k-1}$ is presented by $\langle F \mid \bar{R}\rangle$, where $\bar{R}=\left\langle R, F_{2 k-1}\right\rangle$. Then consider the following commutative diagram where the vertical maps are isomorphisms by Hopf's theorem [Brown 1982, Theorem 5.3, page 42].

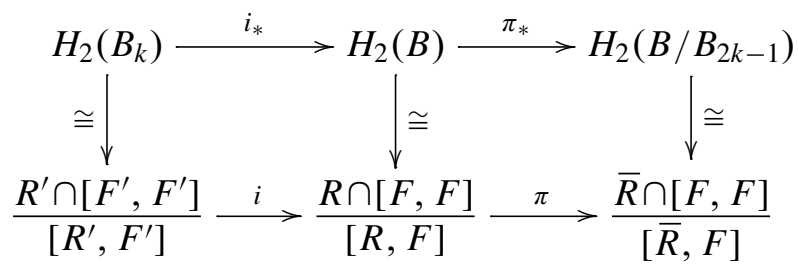

Now note that $i_{*}\left(\left[F^{\prime}, F^{\prime}\right]\right) \subset\left[F_{k}, F_{k}\right] \subset F_{2 k}$. But $F_{2 k}=\left[F_{2 k-1}, F\right] \subset[\bar{R}, F]$. Hence $\pi \circ i=0$ and the result follows.

Finally, $\Gamma_{S, \mathscr{H}}^{n} \subset \Gamma_{S, \mathscr{H}}^{n}$ CH for any class $\mathscr{H}_{\mathbb{Q}}$ contained in $\mathscr{H}_{\mathbb{Q}}^{\mathrm{CH}}$, by Remark 2.8.

We seek to characterize the stabilization of the derived series more precisely. Although the class of maps $\mathscr{H}_{\mathbb{Q}}^{\mathrm{CH}}$ may appear to be the most natural extension to the derived series of Dwyer's class of maps, the following slightly larger class may be even more natural. Indeed, for this class we are able to better characterize the stabilization of the derived series by providing both a "lower bound" and an "upper bound". Both are related to torsion elements of the module $A^{(n)} / A^{(n+1)}$. 
Definition 5.5. For any group $B$ and nonnegative integer, let $\Phi_{H}^{(n)}(B) \subseteq H_{2}(B ; \mathbb{Q})$ be the image of $H_{2}\left(B_{H}^{(n)} ; \mathbb{Q}\right) \rightarrow H_{2}(B ; \mathbb{Q})$, where $B_{H}^{(n)}$ is the torsion-free derived series.

Definition 5.6. Suppose $\mathscr{H}_{H}=\left\{\mathscr{H}_{H}^{(n)}\right\}$, where $\mathscr{H}_{H}^{(n)}$ is the set of homomorphisms $f: A \rightarrow B$, where $A$ is finitely generated, $B$ is finitely related, and $f$ induces an isomorphism on $H_{1}(\cdot ; \mathbb{Q})$ and induces an epimorphism

$$
H_{2}(A ; \mathbb{Q}) / \Phi_{H}^{(n-1)}(A) \rightarrow H_{2}(B ; \mathbb{Q}) / \Phi_{H}^{(n-1)}(B) .
$$

It is easy to see that $\mathscr{H}_{H}$ is closed under composition and, using [Cochran and Harvey 2005, Proposition 2.3], closed under push-outs.

Definition 5.7. Let $A_{C}^{(0)} \equiv A$ and let $A_{C}^{(n+1)}$ be the subgroup generated by the set of elements $x \in A_{C}^{(n)}$ that represent torsion elements of the $\mathbb{Z}\left[A / A_{C}^{(n)}\right]$-module $A_{C}^{(n)} /\left[A_{C}^{(n)}, A_{C}^{(n)}\right]$ that are annihilated by some $\gamma \in \mathbb{Z}\left[A / A_{C}^{(n)}\right]$ whose image under the augmentation $\mathbb{Z}\left[A / A_{C}^{(n)}\right] \rightarrow \mathbb{Z}$ is nonzero.

Proposition 5.8. $\left\{A_{C}^{(n)}\right\}_{n \geq 0}$ is a normal series for $A$ whose successive quotients are $\mathbb{Z}$-torsion free, and $A^{(n)} \subset A_{C}^{(n)} \subset A_{H}^{(n)} \cap A_{2^{n}}^{r}$ for each $n$.

Proof. The proof is by induction on $n$. Suppose the proposition holds for all values less than or equal to $n$. An element $x \in A_{C}^{(n)}$ represents a torsion element as above precisely when there exists $\gamma=\sum k_{i} g_{i} \in \mathbb{Z} A$ such that $\sum k_{i} \neq 0$ and $[x] *[\gamma]=0$, which translates to the condition

$$
\prod_{i} g_{i}^{-1} x^{k_{i}} g_{i} \in\left[A_{C}^{(n)}, A_{C}^{(n)}\right] .
$$

Note that if $x$ satisfies (5-2), then so does $x^{-1}$. A general element of $A_{C}^{(n+1)}$ is a product of such $x$. To show that $A_{C}^{(n+1)}$ is normal in $A$, it suffices to show that $g^{-1} x g$ also satisfies the condition above for any $g \in A$. But this is easily seen by setting $h_{i}=g^{-1} g_{i}$ and observing that

$$
\prod_{i} h_{i}^{-1}\left(g^{-1} x g\right)^{k_{i}} h_{i}=\prod_{i} g_{i}^{-1} x^{k_{i}} g_{i} .
$$

Clearly, $A^{(n+1)} \subset A_{C}^{(n+1)}$ by definition. To show $A_{C}^{(n+1)} \subset A_{H}^{(n+1)}$ assuming that $A_{C}^{(n)} \subset A_{H}^{(n)}$, consider the diagram

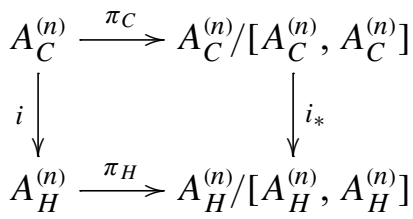

It suffices to show that $i(x) \in A_{H}^{(n+1)}$ for any $x \in A_{C}^{(n)}$ with the property (5-2). But the image $\bar{\gamma}$ of $\gamma$ in $\mathbb{Z}\left[A / A_{H}^{(n)}\right]$ clearly annihilates $\pi_{H}(i(x))$, so it is only necessary 
to remark that $\bar{\gamma} \neq 0$, since it has nonzero augmentation. Thus, $i(x) \in A_{H}^{(n+1)}$ by definition.

To show that $A_{C}^{(n+1)} \subset A_{2^{n+1}}^{r}$, assuming $A_{C}^{(n)} \subset A_{2^{n}}^{r}$, it again suffices to consider a single $x$ satisfying (5-2). We will prove $x \in A_{2^{n+1}}^{r}$ by another induction. Suppose that $x \in A_{m+2^{n}}^{r}$ for some $0 \leq m \leq 2^{n}-1$. Certainly this is true for $m=0$, since

$$
x \in A_{C}^{(n+1)} \subset A_{C}^{(n)} \subset A_{2^{n}}^{r}
$$

by our other inductive hypothesis. Consider our hypothesis

$$
\sum g_{i}^{-1} x^{k_{i}} g_{i} \in\left[A_{C}^{(n)}, A_{C}^{(n)}\right] \subset\left[A_{2^{n}}^{r}, A_{2^{n}}^{r}\right] \subset A_{2^{n+1}}^{r} \subset A_{m+1+2^{n}}^{r}
$$

as a statement in the torsion-free abelian group $A_{m+2^{n}}^{r} / A_{m+1+2^{n}}^{r}$. Since $x \in A_{m+2^{n}}^{r}$,

$$
g_{i}^{-1} x^{k_{i}} g_{i} \equiv x_{i}^{k_{i}} \bmod A_{m+1+2^{n}}^{r}
$$

Thus our hypothesis simplifies to $\left(\sum k_{i}\right) \cdot[x]=0$, implying that $[x]=0$ in this quotient, and hence that $x \in A_{m+1+2^{n}}^{r}$. Iteration yields that $x \in A_{2^{n+1}}^{r}$.

Theorem 5.9. On the class of finitely-presented groups, the stabilization $A_{S}^{(n)}$ of the derived series with respect to the Harvey class of maps $\mathscr{H}_{H}$ satisfies

$$
A_{C}^{(n)} \subset A_{S}^{(n)} \subset A_{H}^{(n)}
$$

(and each of these series is $\mathscr{H}_{H}$-invariant).

Proof. Assume that $A$ is finitely presented. The proof is by induction on $n$. The case $n=0$ is clear since each of the series above is defined to be $A$ itself in that case. Supposing that the theorem holds for all integers less than or equal to $n$, we establish it for $n+1$.

Again we invoke the main theorem of [CH 2008a] (in a stronger form than that used previously).

Theorem 5.10 [CH 2008a, Theorem 2.1]. If $f: A \rightarrow B$ and $f \in \mathscr{H}_{H}^{(n)}$, then $f$ induces a monomorphism

$$
A / A_{H}^{(n+1)} \hookrightarrow B / B_{H}^{(n+1)} .
$$

Corollary 5.11. $A_{H}^{(n+1)}$ is $\mathscr{H}_{H}^{(n-1)}$-invariant (and hence also $\mathscr{H}_{H}^{(n)}$-invariant and $\mathscr{H}_{H}^{(n+1)}$-invariant).

Proof. If $f \in \mathcal{H}_{H}^{(n-1)}, f: A \rightarrow B$, then $f$ induces a monomorphism $A / A_{H}^{(n)} \rightarrow B / B_{H}^{(n)}$ by Theorem 5.10. It then follows from [Cochran and Harvey 2005, Proposition 2.3] that $f\left(A_{H}^{(n+1)}\right) \subset B_{H}^{(n+1)}$. Thus the $(n+1)$-st term of the torsion-free derived series is $\mathscr{H}_{H}^{(n-1)}$-invariant. Since $\mathscr{H}_{H}^{(n+1)} \subset \mathscr{H}_{H}^{(n)} \subset \mathscr{H}_{H}^{(n-1)}$, the other statements follow immediately. 
It follows that the series $\tilde{\Gamma}^{n}(A)=A_{H}^{(n)}$ satisfies properties (3.1) and (3.2) of Theorem 2.10 for $\Gamma^{n}(A)=A^{(n)}$ with respect to $\mathscr{H}_{H}$ and so, by Theorem 2.10, $A_{S}^{(n)} \subseteq A_{H}^{(n)}$.

Now we prove that $A_{C}^{(n+1)} \subset A_{S}^{(n+1)}$, assuming that $A / A_{C}^{(n+1)} \subset A / A_{S}^{(n+1)}$. It suffices to consider $x \in A_{C}^{(n+1)}$ that satisfies (5-2) since a general element is a product of such $x$. Consider the projection map

$$
f: A \rightarrow A /\langle x\rangle \equiv B .
$$

Note that $B$ is also finitely presented. Since $f(x)=1, f(x) \in B^{(n+1)}$. Hence, if we can establish that $f \in \mathscr{H}_{H}^{(n+1)}$, then by definition, $x \in A_{S}^{(n+1)}$ with respect to $\mathscr{H}_{H}$. Since $n \geq 1$, we have $x \in A_{2}^{r}$ by Proposition 5.8, so a multiple of $x$ lies in the commutator subgroup of $A$. Hence $f$ induces an isomorphism on $H_{1}(\cdot ; \mathbb{Q})$. Thus it suffices to show that $f$ induces an epimorphism

$$
H_{2}(A ; \mathbb{Q}) \rightarrow H_{2}(B ; \mathbb{Q}) / \Phi_{H}^{(n)}(B) .
$$

Choose a surjection $\bar{\phi}: \bar{F} \rightarrow A_{H}^{(n)}$, where $\bar{F}$ is free (a generating set for $A_{H}^{(n)}$ ), and extend this to a surjection $\phi: F \rightarrow A$ where $\bar{F} \subset F$ and $F$ is free (a generating set for $A$ ). Then say $A$ is presented by $\langle F \mid R\rangle$ and $B$ is presented by $\langle F \mid R, x\rangle$. The cokernel of $H_{2}(A ; \mathbb{Q}) \rightarrow H_{2}(B ; \mathbb{Q})$ under Hopf's identifications

$$
\frac{R \cap[F, F]}{[R, F]} \otimes \mathbb{Q} \cong H_{2}(A ; \mathbb{Q}) \rightarrow H_{2}(B ; \mathbb{Q}) \cong \frac{\langle R, x\rangle \cap[F, F]}{[\langle R, x\rangle, F]} \otimes \mathbb{Q}
$$

is generated by the class of $x \in F$. Since $g_{i}^{-1} x^{k_{i}} g_{i} \equiv x^{k_{i}}$ modulo $[\langle R, x\rangle, F]$,

$$
y=\prod_{i} g_{i}^{-1} x^{k_{i}} g_{i} \equiv x^{\sum k_{i}} .
$$

Thus $y$ also generates the cokernel of $H_{2}(A ; \mathbb{Q}) \rightarrow H_{2}(B ; \mathbb{Q})$ (under the identifications above). It suffices now to show that $y$ is in the image of $H_{2}\left(B_{H}^{(n)}\right)$. Since $x \in A_{H}^{(n+1)}$ [Cochran and Harvey 2005, Proposition 2.5], the map $f$ above induces an isomorphism $A / A_{H}^{(n)} \cong B / B_{H}^{(n)}$. In particular, this implies that $A_{H}^{(n)} \rightarrow B_{H}^{(n)}$ is surjective. Hence $\bar{F} \rightarrow A_{H}^{(n)} \rightarrow B_{H}^{(n)}$ is surjective and we may suppose that $B_{H}^{(n)} \cong\langle\bar{F} \mid \bar{R}\rangle$. Hence

$$
H_{2}\left(B_{H}^{(n)} ; \mathbb{Q}\right) \cong \frac{\langle\bar{R}\rangle \cap[\bar{F}, \bar{F}]}{[\langle\bar{R}\rangle, \bar{F}]} \otimes \mathbb{Q}
$$

Since by hypothesis

$$
y \in\left[A_{C}^{(n)}, A_{C}^{(n)}\right] \subset\left[A_{H}^{(n)}, A_{H}^{(n)}\right],
$$

$y$ is represented by an element of $[\bar{F}, \bar{F}]$. Moreover, this element is surely in $\bar{R}$, since $y$ represents the trivial element in $B$. Thus the class represented by $y$ is in the image of $H_{2}\left(B_{H}^{(n)}\right)$. 


\section{Relationship with homological localization of groups}

In this section we show that if one considers the class of homologically 2-connected maps, then the stabilization is related to certain known homological localizations. However, this fact does not seem to assist in calculating the stabilization.

Suppose $R=\mathbb{Q}$ or $\mathbb{Z}$, and let $\mathscr{H}^{R}$ be the set of homomorphisms $f: A \rightarrow B$, where $A$ is finitely generated, $B$ is finitely presented, and $f$ induces a 2-connected map on $R$-homology. For any finitely generated group $A$, there exists a group $\hat{A}$ and a functorial assignment $\theta: A \rightarrow \hat{A}$, called the $R$-closure of $A$ [Cha 2008, Theorem 6.1], with the following properties:

(i) For any $f: A \rightarrow B$ in $\mathscr{H}^{R}, f$ induces an isomorphism $\hat{f}: \hat{A} \cong \hat{B}$.

(ii) For any finitely presented group $A$, there is a sequence of finitely presented groups $A_{i}$ and maps $h_{i} \in \mathscr{H}_{R}$

$$
A \stackrel{h_{0}}{\longrightarrow} A_{1} \stackrel{h_{1}}{\longrightarrow} \cdots \stackrel{h_{i-1}}{\longrightarrow} A_{i} \stackrel{h_{i}}{\longrightarrow} \cdots \hat{A},
$$

such that $\lim _{\longrightarrow} A_{i} \cong \hat{A}$.

Proposition 6.1. Let $\left\{\Gamma^{n}\right\}$ be an $\mathscr{H}^{R}$-invariant series. Suppose that $\left\{\Gamma^{n}\right\}$ commutes with direct limits of maps in $\mathscr{H}^{R}$. Then, for any finitely presented group $A$, the stabilization $\Gamma_{S}^{n}(A)$ with respect to $\mathscr{H}^{R}$ is the kernel of

$$
A \stackrel{\theta_{A}}{\longrightarrow} \hat{A} \stackrel{\pi}{\longrightarrow} \hat{A} / \Gamma^{n}(\hat{A}) .
$$

Proof. Let $\widetilde{\Gamma}^{n}(A)$ denote this kernel. We must first verify that $\widetilde{\Gamma}^{n}(A)$ is an $\mathscr{H}^{R}$ invariant series. It will suffice to show that $f\left(\widetilde{\Gamma}^{n}(\hat{A})\right) \subset \widetilde{\Gamma}^{n}(\hat{B})$ for any $f: A \rightarrow B$ in $\mathcal{H}^{R}$. Suppose $a \in \widetilde{\Gamma}^{n}(\hat{A})$. Then $\theta_{A}(a) \in \Gamma^{n}(\hat{A})$. By (6-1) and since $\left\{\Gamma^{n}\right\}$ commutes with direct limits, we have

$$
\Gamma^{n}(\hat{A})=\lim _{\longrightarrow} \Gamma^{n}\left(A_{i}\right),
$$

so there is some $i$ such that $h_{i}(a) \in \Gamma^{n}\left(A_{i}\right)$. Now consider the composition

$$
A_{i} \rightarrow \hat{A} \stackrel{\hat{f}}{\longrightarrow} \hat{B} \text {. }
$$

Since $\hat{B}=\lim _{\longrightarrow} B_{j}$ and $A_{i}$ is finitely generated, there is some $j$ such that this composition factors through $B_{j}$ :

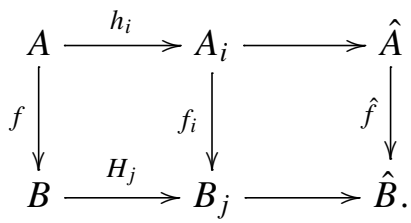


Since $h_{i}, f$ and $H_{j}$ are in $\mathscr{H}^{R}$, we have $f_{i} \in \mathscr{H}^{R}$. Since $\Gamma^{n}$ is $\mathscr{H}^{R}$-invariant, $f_{i}\left(h_{i}(a)\right) \in \Gamma^{n}\left(B_{j}\right)$. Thus $H_{j}(f(a)) \in \Gamma^{n}\left(B_{j}\right)$. Since $\left\{\Gamma^{n}\right\}$ commutes with direct limits of maps in $\mathscr{H}^{R}$,

$$
\Gamma^{n}(\hat{B})=\underline{\lim } \Gamma^{n}\left(B_{j}\right) .
$$

Hence $\theta_{B}(f(a)) \in \Gamma^{n}(\hat{B})$, so $f(a) \in \widetilde{\Gamma}^{n}(\hat{B})$ as desired. Thus $\widetilde{\Gamma}^{n}(A)$ is an $\mathscr{H}^{R}$ invariant series.

By (6-2), we have $\theta_{A}\left(\Gamma^{n}(A)\right) \subset \Gamma^{n}(\hat{A})$, so

$$
\Gamma^{n}(A) \subset \widetilde{\Gamma}^{n}(A),
$$

and therefore $\widetilde{\Gamma}^{n}$ satisfies (3.1) of Theorem 2.10. We next show that $\widetilde{\Gamma}^{n}$ satisfies (3.2) of that theorem. So suppose $f: A \rightarrow B$ lies in $\mathscr{H}^{R}$. By property (i) of the $R$-closure, $\hat{f}$ is an isomorphism. We claim that $\hat{f}\left(\Gamma^{n}(\hat{A})\right) \subset \Gamma^{n}(\hat{B})$. For, if $\alpha \in \Gamma^{n}(\hat{A})$, then as above, $\alpha$ is represented by some $\alpha_{i} \in \Gamma^{n}\left(A_{i}\right)$. The argument above then shows

$$
\hat{f}_{n}: \hat{A} / \Gamma^{n}(\hat{A}) \rightarrow \hat{B} / \Gamma^{n}(\hat{B}) .
$$

It will follow that this map is an isomorphism if we can verify that $(\hat{f})^{-1}$ induces a map on these same quotients (going the other way). This is accomplished by establishing that $(\hat{f})^{-1}$ is necessarily induced by a family of maps $B_{j} \rightarrow A_{i_{j}}$, which are in $\mathscr{H}^{R}$, and then proceeding as above. Therefore $\hat{f}_{n}$ is an isomorphism. Finally, consider $[a]$ in the kernel of

$$
A / \widetilde{\Gamma}^{n}(A) \stackrel{f_{n}}{\longrightarrow} B / \widetilde{\Gamma}^{n}(B) .
$$

By definition, $f(a) \in \widetilde{\Gamma}^{n}(B)$ implies that $\pi_{B}\left(\theta_{B}(f(a))\right)=0$. From the diagram

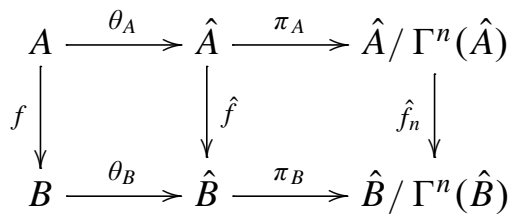

and the fact that $\hat{f}_{n}$ is an isomorphism, it follows that $a \in \widetilde{\Gamma}^{n}(A)$. Thus $f_{n}$ above is a monomorphism and so $\widetilde{\Gamma}^{n}$ satisfies (3.1) of Theorem 2.10. By that theorem then,

$$
\Gamma_{S}^{n}(A) \subset \widetilde{\Gamma}^{n}(A) .
$$

Finally, we show that

$$
\widetilde{\Gamma}^{n}(A) \subset \Gamma_{S}^{n}(A),
$$

which completes our proof that $\widetilde{\Gamma}^{n}=\Gamma_{S}^{n}$. Suppose $a \in \widetilde{\Gamma}^{n}(A)$, so $\theta_{A}(a) \in \Gamma^{n}(\hat{A})$. We saw earlier in the proof that this implies that there is some $i$ such that $h_{i}(a) \in$ $\Gamma^{n}\left(A_{i}\right)$. Since $h_{i} \in \mathscr{H}^{R}$, by definition we have $a \in \Gamma_{S}^{n}(A)$. 


\section{References}

[Bousfield 1977] A. K. Bousfield, Homological localization towers for groups and П-modules, Mem. Amer. Math. Soc. 186, American Mathematical Society, Providence, RI, 1977. MR 56 \#5688 Zbl 0364.20058

[Brown 1982] K. S. Brown, Cohomology of groups, Graduate Texts in Mathematics 87, Springer, New York, 1982. MR 83k:20002 Zbl 0584.20036

[CH 2008a] T. Cochran and S. Harvey, "Homology and derived series of groups, II: Dwyer's theorem”, Geom. Topol. 12:1 (2008), 199-232. MR 2009c:57003 Zbl 1163.20031

[CH 2008b] T. Cochran and S. Harvey, "Homology and derived p-series of groups", J. Lond. Math. Soc. (2) 78:3 (2008), 677-692. MR 2009j:57002 Zbl 1159.57004

[Cha 2008] J. C. Cha, "Injectivity theorems and algebraic closures of groups with coefficients", Proc. Lond. Math. Soc. (3) 96:1 (2008), 227-250. MR 2010b:20092 Zbl 05249002

[Cochran and Harvey 2005] T. Cochran and S. Harvey, "Homology and derived series of groups", Geom. Topol. 9 (2005), 2159-2191. MR 2007c:20120 Zbl 1138.20043

[Dwyer 1975] W. G. Dwyer, "Homology, Massey products and maps between groups", J. Pure Appl. Algebra 6:2 (1975), 177-190. MR 52 \#6710 Zbl 0338.20057

[Harvey 2008] S. L. Harvey, "Homology cobordism invariants and the Cochran-Orr-Teichner filtration of the link concordance group", Geom. Topol. 12:1 (2008), 387-430. MR 2009d:57045 Zbl 1157.57006

[Stallings 1965] J. Stallings, "Homology and central series of groups", J. Algebra 2 (1965), 170181. MR 31 \#232 Zbl 0135.05201

Received June 16, 2008. Revised September 29, 2009.

TIM D. COCHRAN

DEPARTMENT OF MATHEMATiCS, MS 136

RICE UNIVERSITY

PO BOX 1892

Houston, TX 77251-1892

UNITED STATES

cochran@rice.edu

http://math.rice.edu/ cochran

SHELLY HARVEY

DePartment of Mathematics, MS 136

RICE UNIVERSITY

PO BOX 1892

Houston, TX 77251-1892

UNITED STATES

shelly@ rice.edu

http://math.rice.edu/ shelly 